\title{
Integrated face and facial components detection
}

\begin{abstract}
This paper presents an algorithm that detects faces and facial features (eyes, nose and mouth) on images captured by CCTV system under various imaging conditions, such as variation in poses, scale, illumination and occlusion. The system detects face, nose and mouth using three different classifiers, which were created based on the Viola-Jones method [1] and the eyes were detected using an Eye Detection method that consists of resolution reduction, identification of the eye candidates using eye filter [2] and eyes localization based on mean comparison. Experimented on 500 images, the algorithm produced $98.4 \%$ accuracy for face, $98.8 \%$ for nose, $95.6 \%$ for mouth and $94.8 \%$ for eyes.
\end{abstract}

Keyword: Face detection; Facial components detection; Mean comparison; CCTV images 